\title{
Experimental Results of Network-Assisted Interference Suppression Scheme Using Adaptive Beam-Tilt Switching
}

\author{
Tomoki Murakami, ${ }^{1}$ Riichi Kudo, ${ }^{1}$ Koichi Ishihara, ${ }^{1}$ Masato Mizoguchi, ${ }^{1}$ \\ and Naoki Honma ${ }^{2}$ \\ ${ }^{1}$ NTT Access Network Service Systems Laboratories, Nippon Telegraph and Telephone Corporation, Yokosuka, Japan \\ ${ }^{2}$ Faculty of Engineering, Iwate University, Morioka, Japan
}

Correspondence should be addressed to Tomoki Murakami; murakami.tomoki@lab.ntt.co.jp

Received 4 October 2016; Accepted 18 April 2017; Published 14 May 2017

Academic Editor: Ahmed Toaha Mobashsher

Copyright (C) 2017 Tomoki Murakami et al. This is an open access article distributed under the Creative Commons Attribution License, which permits unrestricted use, distribution, and reproduction in any medium, provided the original work is properly cited.

\begin{abstract}
This paper introduces a network-assisted interference suppression scheme using beam-tilt switching per frame for wireless local area network systems and its effectiveness in an actual indoor environment. In the proposed scheme, two access points simultaneously transmit to their own desired station by adjusting angle of beam-tilt including transmit power assisted from network server for the improvement of system throughput. In the conventional researches, it is widely known that beam-tilt is effective for ICI suppression in the outdoor scenario. However, the indoor effectiveness of beam-tilt for ICI suppression has not yet been indicated from the experimental evaluation. Thus, this paper indicates the effectiveness of the proposed scheme by analyzing multiple-input multipleoutput channel matrices from experimental measurements in an office environment. The experimental results clearly show that the proposed scheme offers higher system throughput than the conventional scheme using just transmit power control.
\end{abstract}

\section{Introduction}

To handle the massive mobile traffic from smartphones and tablet devices, data-offloading to small cells such as wireless local area network (WLAN) systems is progressing in addition to the evolution of cellular systems [1-3]. For the efficient operation of the above networks, heterogeneous network (HetNet) with large macrocells in combination with small cells has been researched [4-6]. Small cell technologies in the HetNet have higher potential of the improvement of spectrum efficiency by taking advantage of dense reuse of frequency channel [7-9]. However, as excess installations of small cell cause intercell interference (ICI), it is difficult to obtain the maximum effect of frequency channel reuse [10].

To suppress ICI between small cells, network-assisted transmit power control (TPC) technologies, which cooperatively suppress ICI by adjusting each transmit power at multiple APs and improve system throughput, have been investigated [11, 12]; unfortunately, they also degrade the received power at each station (STA) because of TPC. More recently, network-assisted beamforming technologies also have been investigated [13-16]. They cooperatively suppress ICI without significantly degrading the received power at each STA except for cases in which the correlation of propagation channels between AP and desired/nondesired STAs is high. Moreover, beamforming techniques including TPC have been proposed and its effectiveness is clarified in [17].

Beamforming technologies on orthogonal frequency division multiplexing (OFDM) are classified into two categories: digital beamforming, which controlled amplitude and phase per subcarrier by digital signal processing, and analog beamforming, which controlled them in common for all subcarriers by using phase shifters connected to each antenna. Digital beamforming offers higher capacity and greater flexibility because of its precise control per subcarrier $[13,14]$. However, it demands precise frequency/time synchronizations and large computational load for transmission weight calculation. On the other hand, analog beamforming is done by using phase shifters $[15,16]$. While it dispenses with digital signal processing units, its throughput performance is less than that of digital beamforming because beamforming is common to all subcarriers. More recently, techniques that 


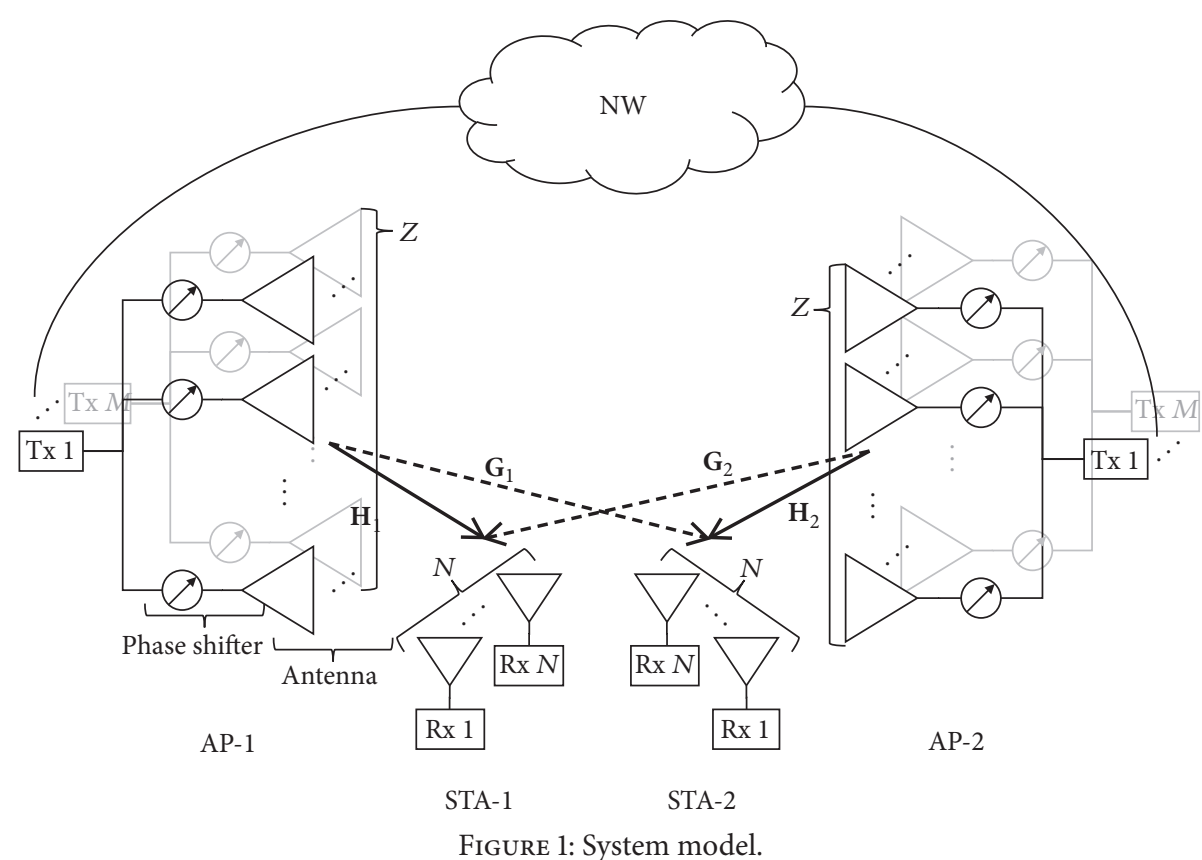

attempt to offer the features of both digital and analog beamforming have been investigated [18-20]. Considering the future wireless system, it is necessary to reevaluate each technology of both analog and digital beamforming. This paper focuses on beam-tilt technologies as analog beamforming for ICI suppression.

In the conventional researches, it is widely known that beam-tilt is effective for ICI suppression in the outdoor scenario such as macrocells. In the outdoor scenario, since the influence of direct wave path is relatively strong, ICI is expected to be greatly suppressed by avoiding direct wave path [15]. Also in the indoor scenario, although the effect of ICI suppression becomes smaller than that in the outdoor scenario because of multipath environment, there are reports which indicate the effectiveness of beam-tilt using the frequency band of WLAN systems. For example, [21] presents the multiple-input multiple-output (MIMO) antenna design including beam-tilt and its effectiveness for indoor base stations but does not mention the effect of ICI suppression from experimental evaluations.

In this paper, we introduce a network-assisted interference suppression scheme using adaptive beam-tilt switching including TPC for WLAN systems. In the proposed scheme, two APs simultaneously transmit to their own desired STA by adjusting angle of beam-tilt and transmit power per frame according to STA locations for the improvement of system throughput. As an additional technique, the proposed scheme uses a simple setting approach, which calculates angle of beam-tilt from channel state information (CSI) between the selected horizontal row of AP antenna elements and STA, given that there are fewer radio frequency (RF) front ends than antenna elements. In order to clarify the effectiveness of our adaptive beam-tilt switching in an indoor environment, we have evaluated the proposed scheme by computer simulations based on raytracing [22]. For the next step toward practical use, we evaluate the proposed scheme by using measured MIMO channel matrices in an indoor office environment. The experimental results clearly show that the proposed scheme offers higher system throughput than the conventional power control.

This paper is organized as follows. Section 2 introduces the system model. Section 3 describes our proposed interference suppression scheme. Section 4 details a performance analysis that uses the measured MIMO channel matrices. We conclude with a summary of key points in Section 5 .

Notations. Vectors and matrices are in boldface. Superscript $\mathbf{A}^{H}$ denotes the Hermitian transpose operator of $\mathbf{A}, \mathbf{A}^{-1}$ denotes the inverse of $\mathbf{A},\|\mathbf{A}\|_{F}$ denotes the Frobenius norm of $\mathbf{A}, \mathbf{I}_{N}$ is an identity matrix of size $(N \times N), \mathbb{C}^{N \times M}$ denotes the set of complex matrix of size $(N \times M)$, $\operatorname{det}(\mathbf{A})$ is the determinant of $\mathbf{A}$, and $\varepsilon\{\mathbf{A}\}$ is expectation of $\mathbf{A}$.

\section{System Model}

Figure 1 shows the system model in this paper; two APs simultaneously transmit to their own desired STA on downlink MIMO-OFDM transmission. APs and STAs utilize the same frequency channel and bandwidth. It is also assumed that each AP has an array of $M \times Z$ antenna elements ( $M$ : the number of horizontal rows, $Z$ : the number of vertical columns) for communication with its own desired STA equipped with $N$ antennas $(M \geq N)$. $Z$ antenna elements control angle of beam-tilt via analog beamforming by using phase shifters connected to each antenna element. On the other hand, $M$ antenna elements perform MIMO transmission via digital beamforming. It is assumed that transmit time and center frequency are synchronized at both APs. Figure 2 shows the conceptual design of beam-tilt when $Z$ antenna elements are spaced by $d_{z}$. When angle of beam-tilt 
is $\theta$, phase difference $\phi$ between antenna elements is expressed by

$$
\phi=\frac{2 \pi d_{z} \sin \theta}{\lambda},
$$

where $\lambda$ is wavelength. $\mathbf{H}_{k}^{1 \rightarrow 1}\left(\phi_{1}\right), \mathbf{H}_{k}^{2 \rightarrow 2}\left(\phi_{2}\right) \in \mathbb{C}^{N \times M}$ denote MIMO channel matrices of the $k$ th $(k=1, \ldots, K)$ subcarrier between AP-1 and STA-1, AP-2, and STA-2 after adjusting angle of beam-tilt and are expressed by

$$
\begin{aligned}
\mathbf{H}_{k}^{1 \rightarrow 1}\left(\phi_{1}\right) & \\
= & {\left[\sum_{z=1}^{Z} \frac{e^{j \phi_{1}(z-1)}}{\sqrt{Z}} \mathbf{h}_{1,1, z, k}^{\prime} \cdots \sum_{z=1}^{Z} \frac{e^{j \phi_{1}(z-1)}}{\sqrt{Z}} \mathbf{h}_{1, M, z, k}^{\prime}\right], } \\
\mathbf{H}_{k}^{2 \rightarrow 2}\left(\phi_{2}\right) & \\
= & {\left[\sum_{z=1}^{Z} \frac{e^{j \phi_{2}(z-1)}}{\sqrt{Z}} \mathbf{h}_{2,1, z, k}^{\prime} \cdots \sum_{z=1}^{Z} \frac{e^{j \phi_{2}(z-1)}}{\sqrt{Z}} \mathbf{h}_{2, M, z, k}^{\prime}\right], }
\end{aligned}
$$

where $\mathbf{h}_{1, M, z, k}^{\prime}, \mathbf{h}_{2, M, z, k}^{\prime} \in \mathbb{C}^{N \times 1}$ denote channel vectors of the $k$ th subcarrier between ( $m$ th $(m=1, \ldots, M), z$ th $(z=1, \ldots, Z))$ antenna element of AP-1 and STA-1 and ( $m$ th, $z$ th) antenna element of AP-2 and STA-2, respectively. Also, $\phi_{1}, \phi_{2}$ are parameters of beam-tilt of AP-1 and AP-2, respectively. $\mathbf{G}_{k}^{1 \rightarrow 2}\left(\phi_{1}\right), \mathbf{G}_{k}^{2 \rightarrow 1}\left(\phi_{2}\right) \in \mathbb{C}^{N \times M}$ denote MIMO channel matrices between AP-1 and STA-2 and AP-2 and STA-1 after adjusting angle of beam-tilt and are expressed by

$$
\begin{aligned}
& \mathbf{G}_{k}^{1 \rightarrow 2}\left(\phi_{1}\right) \\
& =\left[\sum_{z=1}^{Z} \frac{e^{j \phi_{1}(z-1)}}{\sqrt{Z}} \mathbf{g}_{1,1, z, k}^{\prime} \cdots \sum_{z=1}^{Z} \frac{e^{j \phi_{1}(z-1)}}{\sqrt{Z}} \mathbf{g}_{1, M, z, k}^{\prime}\right], \\
& \mathbf{G}_{k}^{2 \rightarrow 1}\left(\phi_{2}\right) \\
& =\left[\sum_{z=1}^{Z} \frac{e^{j \phi_{2}(z-1)}}{\sqrt{Z}} \mathbf{g}_{2,1, z, k}^{\prime} \cdots \sum_{z=1}^{Z} \frac{e^{j \phi_{2}(z-1)}}{\sqrt{Z}} \mathbf{g}_{2, M, z, k}^{\prime}\right],
\end{aligned}
$$

where $\mathbf{g}_{1, M, z, k}^{\prime}, \mathbf{g}_{2, M, z, k}^{\prime} \in \mathbb{C}^{N \times 1}$ denote channel vectors of the $k$ th subcarrier between ( $m$ th, $z$ th) antenna element of AP-1 and STA- 2 and ( $m$ th, $z$ th) antenna element of AP-2 and STA1 , respectively. The received signals of the $k$ th subcarrier, $\mathbf{y}_{1, k}$, $\mathbf{y}_{2, k} \in \mathbb{C}^{M \times 1}$ at STA-1 and STA-2, are expressed by transmit signals of the $k$ th subcarrier, $\mathbf{x}_{1, k}, \mathbf{x}_{2, k} \in \mathbb{C}^{N \times 1}$ at AP-1, AP-2,

$$
\begin{aligned}
\mathbf{y}_{1, k}= & \sqrt{\frac{p_{1}}{N}} \mathbf{H}_{k}^{1 \rightarrow 1}\left(\phi_{1}\right) \mathbf{x}_{1, k}+\sqrt{\frac{p_{2}}{N}} \mathbf{G}_{k}^{2 \rightarrow 1}\left(\phi_{2}\right) \mathbf{x}_{2, k} \\
& +\mathbf{n}_{1, k}, \\
\mathbf{y}_{2, k}= & \sqrt{\frac{p_{2}}{N}} \mathbf{H}_{k}^{2 \rightarrow 2}\left(\phi_{2}\right) \mathbf{x}_{2, k}+\sqrt{\frac{p_{1}}{N}} \mathbf{G}_{k}^{1 \rightarrow 2}\left(\phi_{1}\right) \mathbf{x}_{1, k} \\
& +\mathbf{n}_{2, k},
\end{aligned}
$$

where $\mathbf{n}_{1, k}, \mathbf{n}_{2, k}$ are additive white Gaussian noise vectors of the $k$ th subcarrier with variance $\sigma^{2} . p_{1}, p_{2}$ are transmit powers at AP-1 and AP-2 $\left(p_{0} \geq p_{1}, p_{2}\right)$, respectively. $p_{0}$ is the maximum transmit power.

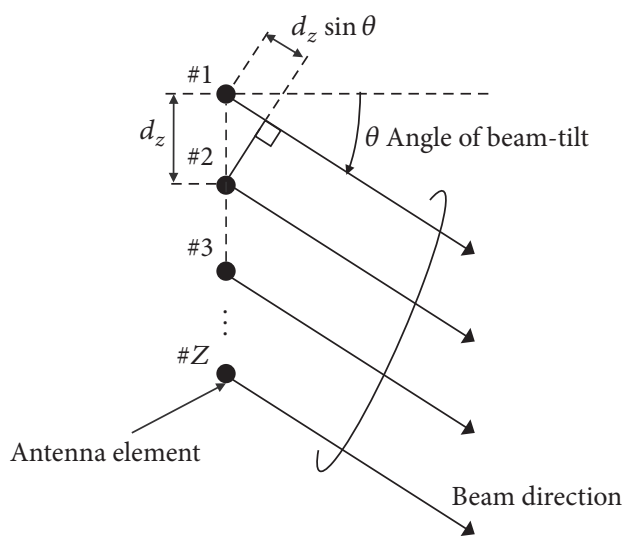

FIgURE 2: Conceptual design of beam-tilt.

\section{Proposed Scheme}

This section explains the network-assisted interference suppression scheme using adaptive beam-tilt switching including TPC. In the proposed scheme, two APs simultaneously transmit to their own desired STA on MIMO-OFDM transmission while adjusting parameters (angle of beam-tilt and transmit power) calculated to suit STA locations. Moreover, the proposed scheme can be applied to environments with many APs and STAs by generating AP pairs with STAs. The proposed scheme uses two setting approaches to adjust those parameters by using CSI. First approach (optimal) uses all CSI for the maximization of system throughput and another approach (simple) uses only partial CSI for the overhead reduction in CSI acquisition. In the optimal approach, although maximum overhead for CSI acquisition (training signal (TS) transmission, CSI feedback, and so on) is required, its transmission capacity except for overhead is expected to be greatly high. On the other hand, the simple approach reduces the overhead for CSI acquisition by calculating parameters from partial CSI by focusing on the selected horizontal row of antenna elements. Figure 3 shows an example frame sequence (trigger frame, CSI acquisition, parameter notice, and data transmission) in order to realize the proposed scheme. Figure 4 shows the process of TS transmission $(i(i=1, \ldots, I)$ is the number of TS transmissions) when there are fewer RF front ends than antenna elements. In the first step, network server informs the start of cooperation to APs via a trigger frame including information of target APs and STAs. In the second step, AP-1 transmits TSs to STA-1 and STA-2 while changing the antenna element to RF front end connection as shown in Figure 4. Each STA estimates CSI from TSs and then feedbacks CSI to AP-1 via CSI feedback (CSI-FB) frame. AP- 2 also acquires CSI by using the same process. In the next step, after AP-1 and AP-2 pass acquired CSI to network server via a CSI notification (CSIN) frame, network server calculates angles of beam-tilt and transmit powers of AP-1 and AP-2 that improve the system throughput and then passes calculated parameters to AP-1 and AP-2 via an angle and power notification (AP-N) frame. In the final step, APs simultaneously transmit data packets 


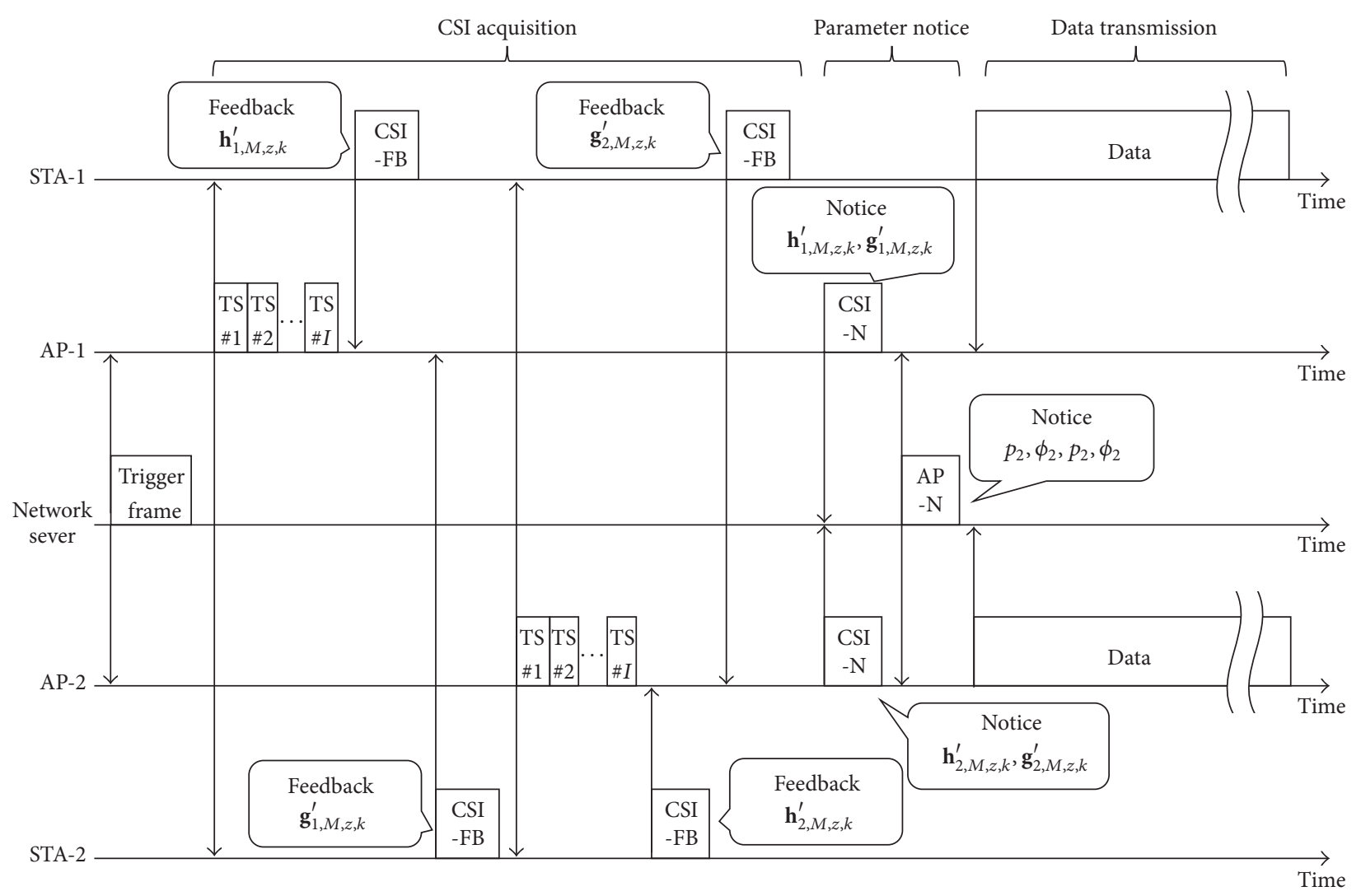

TS: training signal, CSI-FB: CSI feedback,

CSI-N: CSI notice, AP-N: angle and power notice

Figure 3: Frame sequence.

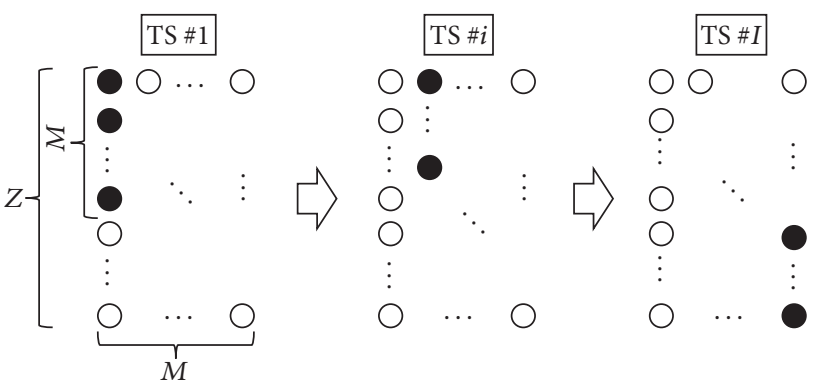

Antenna element connected to RF front-end Antenna element

Figure 4: TS transmission process.

to their own desired STA after each AP sets angle of beamtilt and transmit power by using configuration as shown in Figure 1.

\subsection{Definition of System Throughput in the Optimal Approach.} The optimal approach calculates the system throughput by the exhaustive search with parameters (angle and transmit power) from (5) and CSI in the actual environment. Therefore, the system throughput is maximized for the experimental environment. It is assumed that transmit powers are $p_{1}, p_{2}$ and angles of beam-tilt are $\phi_{1}, \phi_{2}$ at AP-1 and AP-2, respectively. The system throughput, $C$, by adjusting angle of beam-tilt and transmit power is given by

$$
\begin{aligned}
& C=\sum_{k=1}^{K}\left(C_{1, k}\left(\phi_{1}, \phi_{2}, p_{1}, p_{2}\right)+C_{2, k}\left(\phi_{1}, \phi_{2}, p_{1}, p_{2}\right)\right), \\
&\left(\phi_{1}, \phi_{2}, p_{1}, p_{2}\right)=\underset{\phi_{1}, \phi_{2}, p_{1}, p_{2}}{\arg \max }\left(\sum _ { k = 1 } ^ { K } \left(C_{1, k}\left(\phi_{1}, \phi_{2}, p_{1}, p_{2}\right)\right.\right. \\
&\left.\left.\quad+C_{2, k}\left(\phi_{1}, \phi_{2}, p_{1}, p_{2}\right)\right)\right),
\end{aligned}
$$

where $C_{1, k}\left(\phi_{1}, \phi_{2}, p_{1}, p_{2}\right), C_{2, k}\left(\phi_{1}, \phi_{2}, p_{1}, p_{2}\right)$ denote transmission rates of STA-1, STA- 2 on the $k$ th subcarrier calculated by

$$
\begin{gathered}
C_{1, k}\left(\phi_{1}, \phi_{2}, p_{1}, p_{2}\right)=\log _{2} \operatorname{det}\left(\mathbf{I}_{N}+\frac{p_{1}}{N} \mathbf{H}_{k}^{1 \rightarrow 1}\left(\phi_{1}\right)\right. \\
\left.\cdot \mathbf{H}_{k}^{1 \rightarrow 1}\left(\phi_{1}\right)^{H}\left(\mathbf{I}_{N}+\frac{p_{2}}{N} \mathbf{G}_{k}^{2 \rightarrow 1}\left(\phi_{2}\right) \mathbf{G}_{k}^{2 \rightarrow 1}\left(\phi_{2}\right)^{H}\right)^{-1}\right) \\
C_{2, k}\left(\phi_{1}, \phi_{2}, p_{1}, p_{2}\right)=\log _{2} \operatorname{det}\left(\mathbf{I}_{N}+\frac{p_{2}}{N} \mathbf{H}_{k}^{2 \rightarrow 2}\left(\phi_{2}\right)\right. \\
\left.\cdot \mathbf{H}_{k}^{2 \rightarrow 2}\left(\phi_{2}\right)^{H}\left(\mathbf{I}_{N}+\frac{p_{1}}{N} \mathbf{G}_{k}^{1 \rightarrow 2}\left(\phi_{1}\right) \mathbf{G}_{k}^{1 \rightarrow 2}\left(\phi_{1}\right)^{H}\right)^{-1}\right) .
\end{gathered}
$$




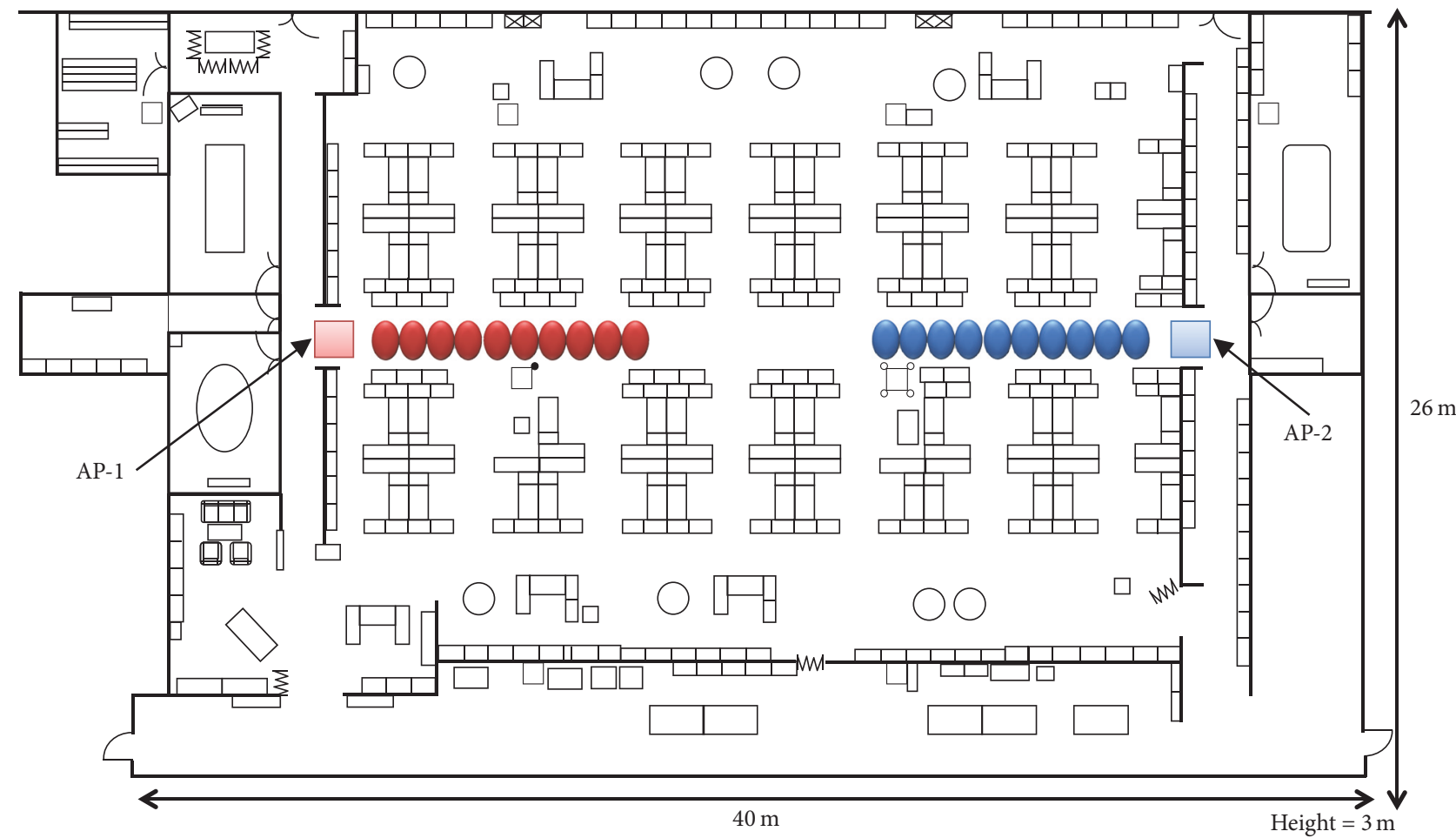

Location of STAs connected to AP-1

Location of STAs connected to AP-2

FIGURE 5: Measurement environment.

3.2. Definition of System Throughput in the Simple Approach. As shown in Figure 4, the simple approach calculates the suboptimal angle of beam-tilt from partial CSI between AP antenna elements on a randomly selected horizontal row and STA. Therefore, overhead for CSI acquisition and required number of RF front ends are greatly decreased. Using AP antenna element on the $m$ th row, values of $\left(\phi_{1}, \phi_{2}, p_{1}, p_{2}\right)$ that improves the system throughput are given by

$$
\begin{aligned}
& \left(\phi_{1}, \phi_{2}, p_{1}, p_{2}\right)=\underset{\phi_{1}, \phi_{2}, p_{1}, p_{2}}{\arg \max }\left(\sum_{k=1}^{K} \widehat{C}_{1, k}\left(\phi_{1}, \phi_{2}, p_{1}, p_{2}\right)\right. \\
& \left.+\widehat{C}_{2, k}\left(\phi_{1}, \phi_{2}, p_{1}, p_{2}\right)\right),
\end{aligned}
$$

where $\widehat{C}_{1, k}\left(\phi_{1}, \phi_{2}, p_{1}, p_{2}\right), \widehat{C}_{2, k}\left(\phi_{1}, \phi_{2}, p_{1}, p_{2}\right)$ denote transmission rates of STA-1, STA- 2 on the $k$ th subcarrier and the $m$ th row of AP antenna element.

\section{Experimental Analysis}

4.1. Experimental Environment. To clarify the effectiveness of the proposed scheme in the indoor static line-of-sight (LOS) environment, which demonstrates little network latency, we previously measured MIMO-OFDM channel matrices in our office (shown in Figure 5) by using our MIMO-OFDM testbed [23] based on IEEE802.11n standard. The height, width, and depth of our office are 3, 40, and $26 \mathrm{~m}$, respectively.
AP-1 and AP-2 set at opposite sides of our office as shown in Figure 5 for the purpose of evaluation and comparing large interference environments. Additionally, the existing office's APs are placed in the same positions. We measured MIMO-OFDM channel matrices at many STA locations, which allow evaluation of a variety of interference states; see Figure 5. Note that the measured channel matrices include the effect of reflection from the concreate walls and metal lockers in our office. The configuration and parameters of our MIMO-OFDM testbed are shown in Figure 6 and Table 1 . The transmitter transmits training signals including short and long preambles and the receiver estimates CSI from training signals. The measurements were centered at $4.85 \mathrm{GHz}$ (matches our experimental wireless license). The bandwidth and the number of subcarriers are $20 \mathrm{MHz}$ and 52, respectively. AP has two horizontal rows and eight vertical columns of patch antennas with vertical element spacing of 0.5 wavelengths. STA has two dipole antennas with horizontal element spacing of 0.5 wavelengths. The heights of AP and STA are 3 and $1 \mathrm{~m}$, respectively. Figures 7(a)-7(f) show photographs at AP and STA antenna configurations and radiation patterns at $4.85 \mathrm{GHz}$, respectively. The total transmit power of each AP is $13 \mathrm{dBm}$.

4.2. Analysis of Beam-Tilt Switching. In this subsection, we indicate the effectiveness of beam-tilt switching and relevance of the experimental environment from the comparison between simulation and experimental results. 


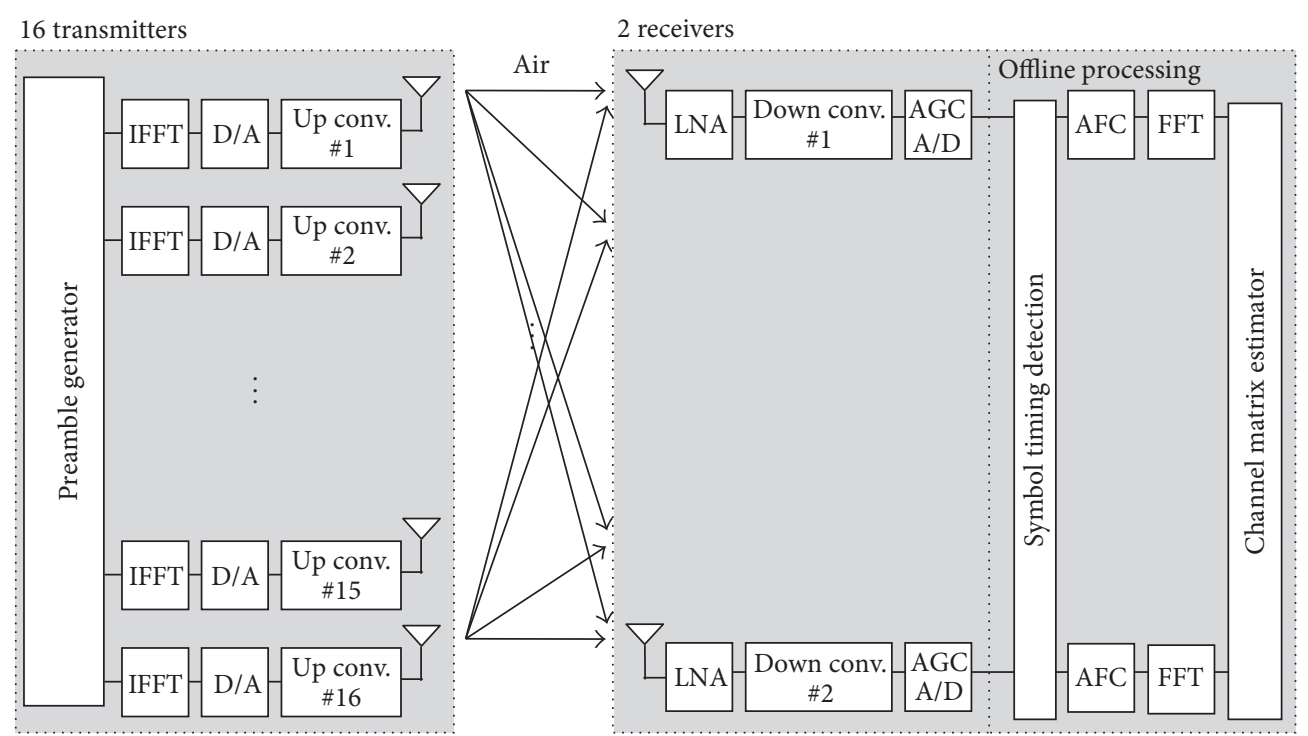

FIGURE 6: Configuration of MIMO-OFDM testbed.

TABLE 1: Measurement parameters.

\begin{tabular}{lc}
\hline Parameter & Value \\
\hline Carrier frequency & $4.85 \mathrm{GHz}$ \\
Bandwidth (subcarrier number) & $20 \mathrm{MHz}(52)$ \\
Transmit power per AP & $13 \mathrm{dBm}$ \\
AP & \\
Antenna & Patch \\
Gain & $9 \mathrm{dBi}$ \\
Antenna element number per row & 2 \\
Antenna element number per column & 8 \\
Antenna element spacing & $0.5 \lambda$ \\
Antenna height & $3 \mathrm{~m}$ \\
STA & \\
Antenna & Dipole \\
Gain & $2 \mathrm{dBi}$ \\
Antenna element number per row & 2 \\
Antenna element number per column & 1 \\
Antenna element spacing & $0.5 \lambda$ \\
Antenna height & $1 \mathrm{~m}$ \\
Beam-tilt-angle & $0-50 \mathrm{deg}$. \\
Transmit power attenuation & $0-50 \mathrm{~dB}$ \\
\hline
\end{tabular}

First of all, Figures 8(a)-8(f) plot the signal to noise power ratio (SNR) distributions when AP-1 uses the beamtilt-angles of $0 \sim 50$ degrees in the indoor simulation model via ray-trace. In this figure, we assume that dimensions are $40 \mathrm{~m}$ (long), $20 \mathrm{~m}$ (wide), and $3 \mathrm{~m}$ (high). Also the ceiling and side walls are made of $15 \mathrm{~cm}$ thick concrete. STA are set in a lattice pattern per $\mathrm{m}^{2}$ in this area. The complex relative permittivity of concrete is $6-j 0.2$ and in all simulation the reflection number is 4 times. The noise power at STA is $-90 \mathrm{dBm}$. Other parameters are the same as the experimental value as shown in Table 1. From this figure, we found that SNR distribution is greatly changed by the value of beam-tiltangle. In other words, interference power from other AP is also greatly changed.

On the other hand, Figure 9 shows SNR between AP-1, AP-2, and their own desired STA at the limited area as shown in Figure 5 versus angle of beam-tilt in the indoor experimental environment. SNR in this evaluation is the average value of SNR at each STA. From this result, it is found that SNR is maximized when angles of beam-tilt are set about 30 degrees in this environment. In addition, SNR is improved when the number of antenna elements increases. Concretely, SNR with beam-tilt is $1(Z=2), 5(Z=4)$, and $7(Z=8) \mathrm{dB}$ higher than that without beam-tilt. Next, Figure 10 shows interference signal to noise power ratio (INR) between AP-1, AP-2, and nondesired STA versus angle of beam-tilt. Similar to Figure 8, INR is average value of INR at each STA. From this result, it is found that beam-tilt performs SNR improvement and ICI suppression even if in indoor environment. Figure 11 shows the SNR between AP-1 and all of STAs when beam-tilt angles were set to $0,10,20,30,40$, and 50 degrees. Similar to results of Figures 9 and 10, beam-tilt changes the SNR performance. Moreover, we recognized that the tendency of the experimental result (Figure 8) and the simulation result (Figure 11) is close. Therefore, we consider that it is possible to evaluate the effectiveness of the proposed scheme by using the experimental evaluations in the limited area.

4.3. Analysis of the Proposed Scheme. Figure 12 shows the cumulative distribution function (CDF) of system throughput performances of adaptive beam-tilt switching, TPC, and both (proposal) with optimal and simple approaches. The plots cover the system throughputs for all STA locations. The $\mathrm{CDF}$ of the maximum power transmission at APs is also plotted as the conventional scheme. In this evaluation, we assume that AP has eight antenna elements $(Z=8)$ and varies 


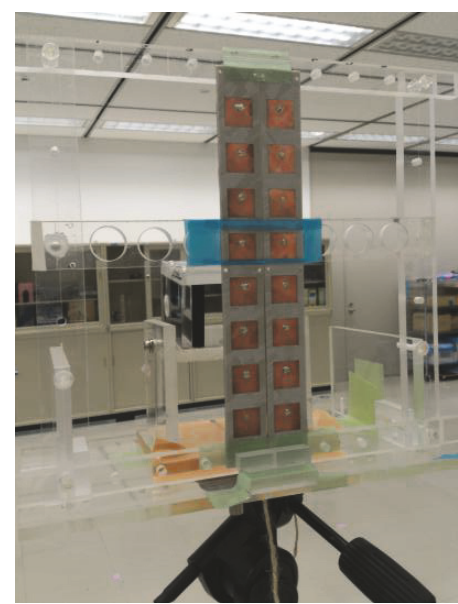

(a) AP antenna

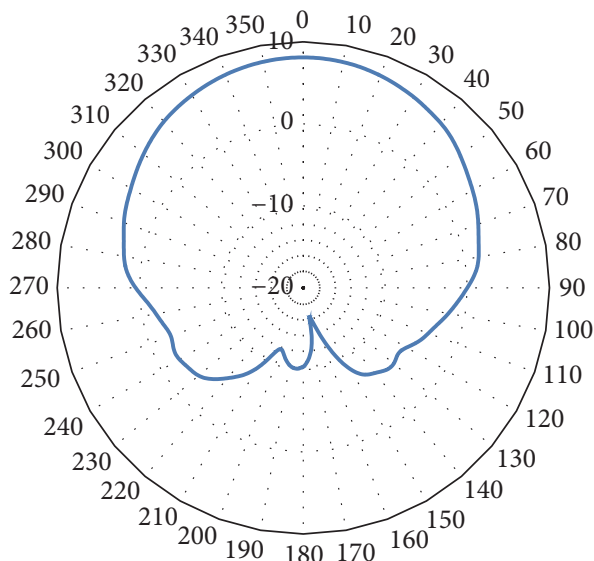

(c) Radiation pattern of AP antenna (E-plane)

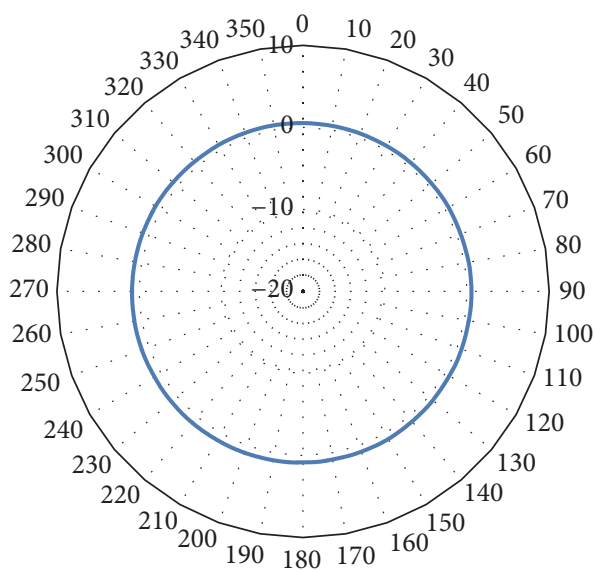

(e) Radiation pattern of STA antenna (E-plane)

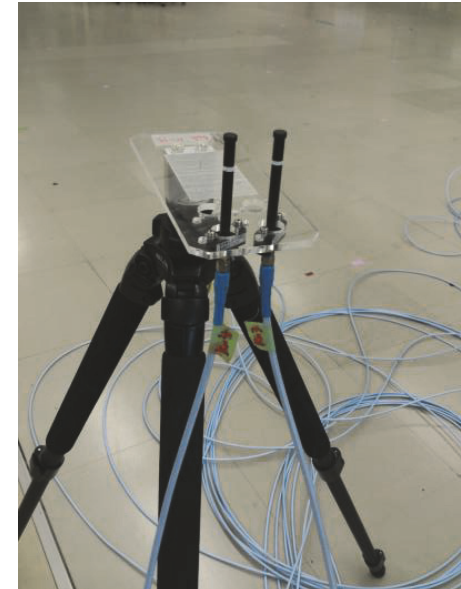

(b) STA antenna

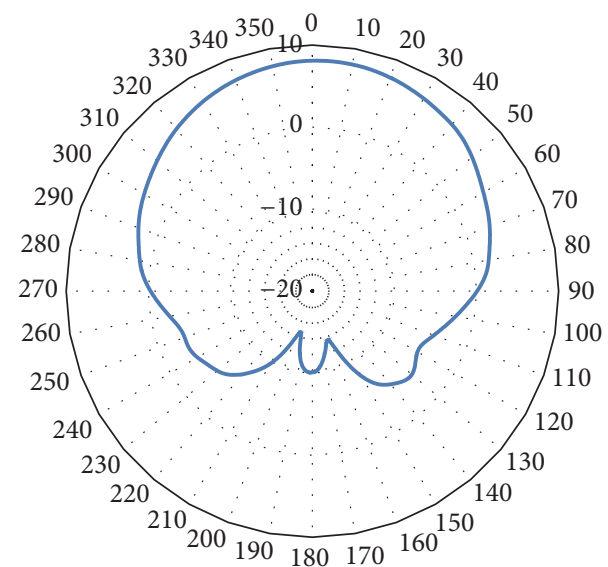

(d) Radiation pattern of AP antenna (H-plane)

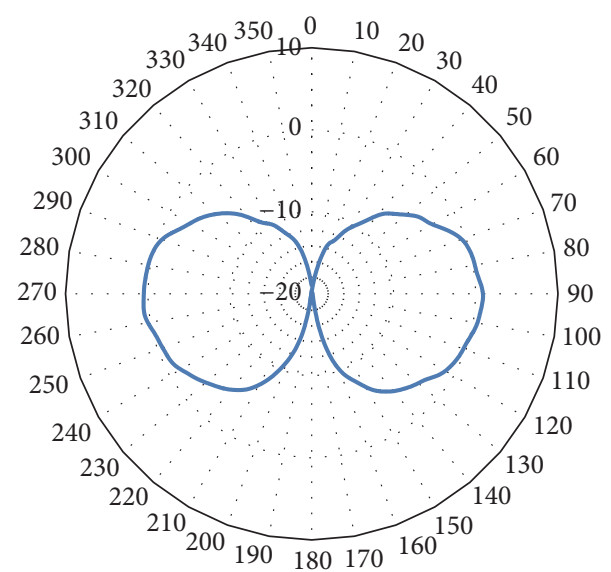

(f) Radiation pattern of STA antenna (H-plane)

FIGURE 7: Photographs and radiation patterns of AP and STA antennas.

angle of beam-tilt from 0 to 50 degrees in 5-degree steps, with transmission power attenuation from 0 to $50 \mathrm{~dB}$ in $5 \mathrm{~dB}$ steps. From the results, improvements of system throughput $50 \%$ CDF value) by using beam-tilt, power, and both controls are $2.8,2.0$, and 3.78 times higher than that of the maximum power transmission, respectively. Since the distance between APs is short in this environment (in other words, when ICI is large), the effectiveness of beam-tilt control is less than that of TPC. However, combining beam-tilt switching and TPC offers higher system throughput than just TPC. 


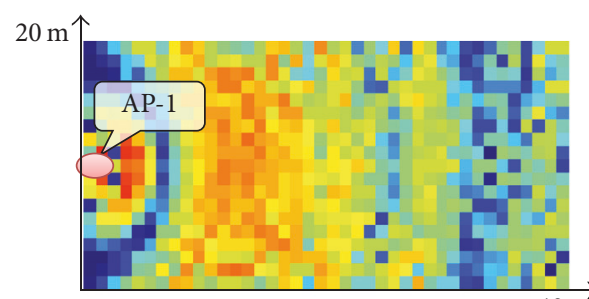

(a) $\theta=0$ deg.

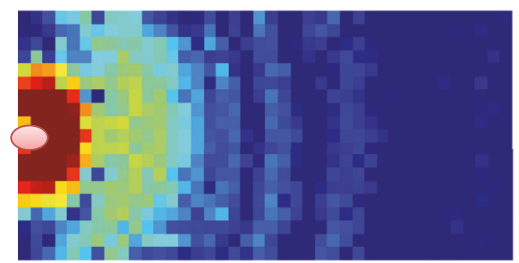

(d) $\theta=30 \mathrm{deg}$.

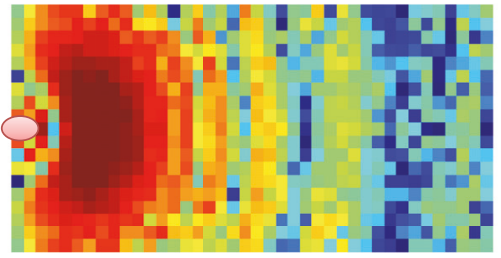

(b) $\theta=10 \mathrm{deg}$.

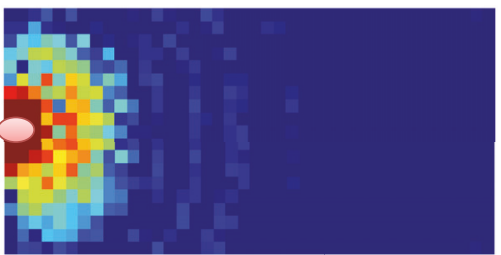

(e) $\theta=40 \mathrm{deg}$.

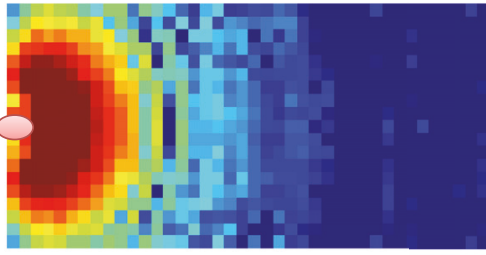

(c) $\theta=20 \mathrm{deg}$.

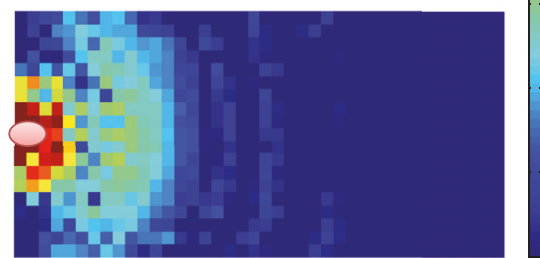

(f) $\theta=50$ deg.

FIGURE 8: SNR distribution when $\theta$ of AP-1 is set from 0 to 50 degrees.

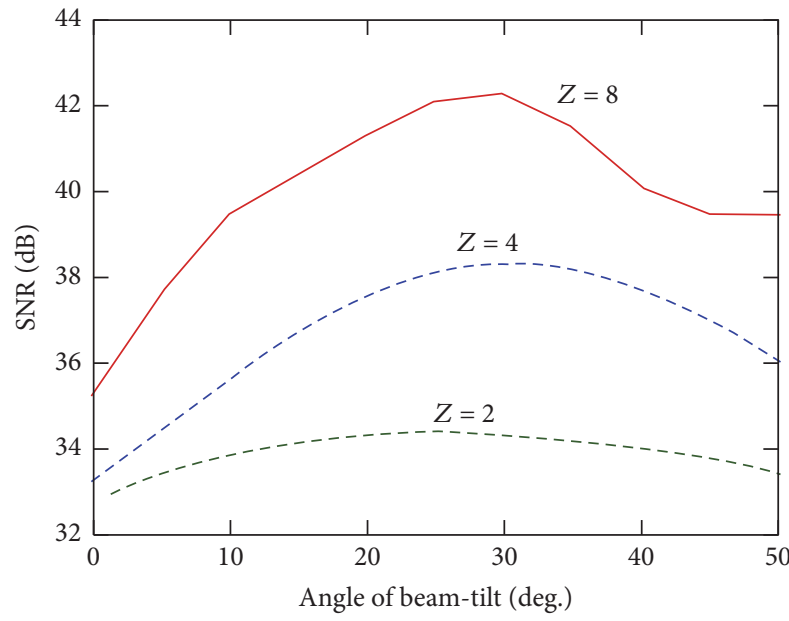

FIGURE 9: SNR versus angle of beam-tilt.

Figure 13 shows system throughput performances of the proposed scheme based on adaptive beam-tilt with TPC (10 and 50\% CDF values) versus the column number of AP antenna elements. This result shows that the system throughputs on both optimal and simple approaches achieve increase with the column number of AP antenna elements. This is because SNR at the desired STA improves as the array gain increases. Then, system throughput with the simple approach is less than that with optimal approach because only one array column is selected from all rows at random. However, the system throughput penalty (50\% value at CDF) by using the simple approach is so small. If this penalty is acceptable, the proposed scheme with the simple approach offers a significant reduction in CSI feedback overhead, the number of RF front ends, and calculation loads.

\section{Conclusions}

In this paper, we experimentally evaluated the network-assisted interference suppression scheme using adaptive beamtilt switching including TPC for WLAN systems. Also the

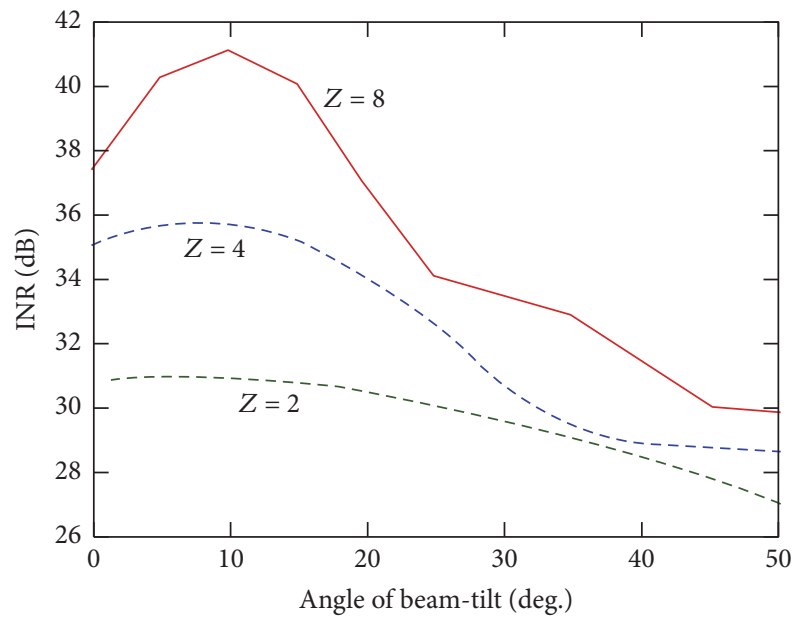

FIGURE 10: INR versus angle of beam-tilt.

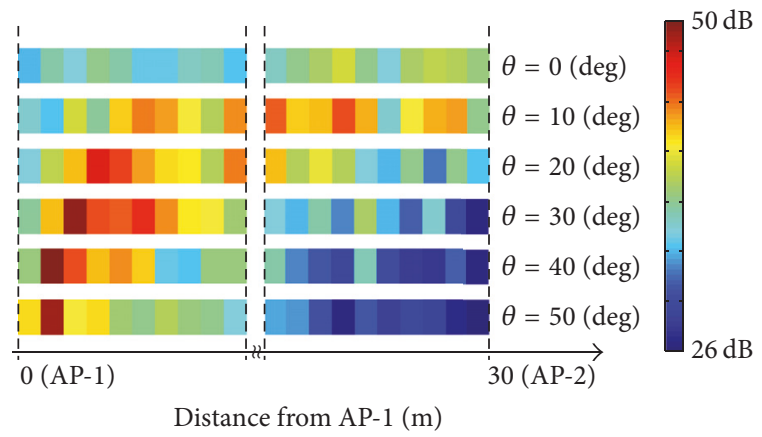

FIGURE 11: SNR distribution per angle of beam-tilt $(Z=8)$.

proposed scheme has optimal and simple setting approaches for adaptive beam-tilt switching. Moreover, we introduced the frame sequence and CSI estimation method for implementation of the proposed scheme in WLAN systems. In order to clarify the effectiveness of the proposed scheme, we evaluated the proposed scheme by using MIMO channel 


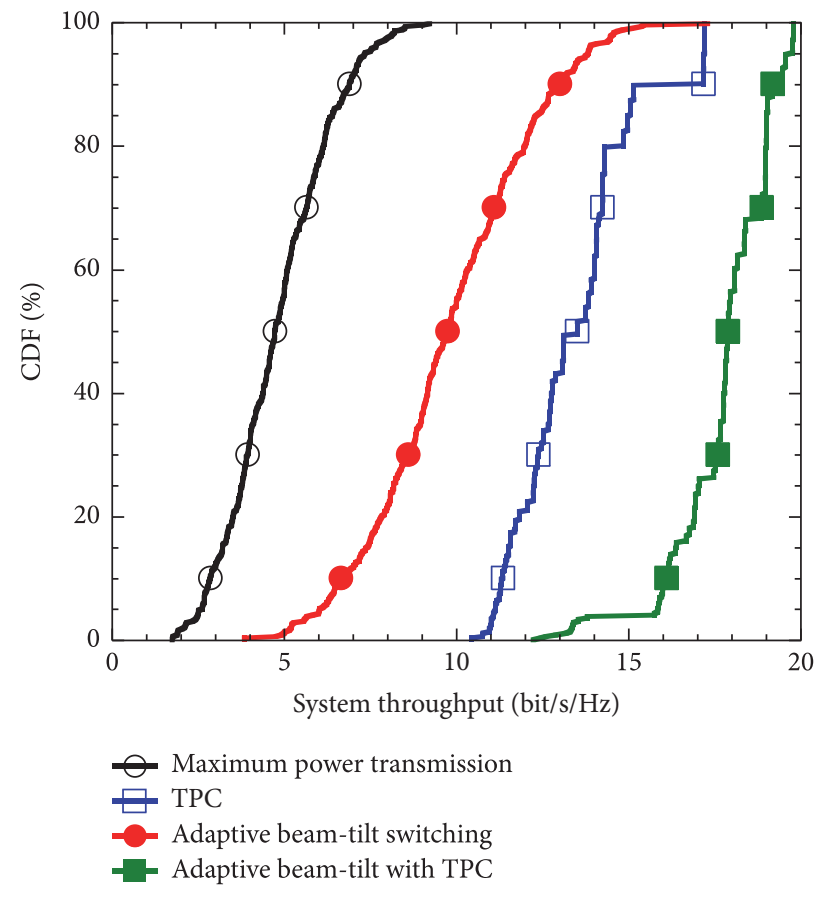

FIGURE 12: CDF of system throughput.

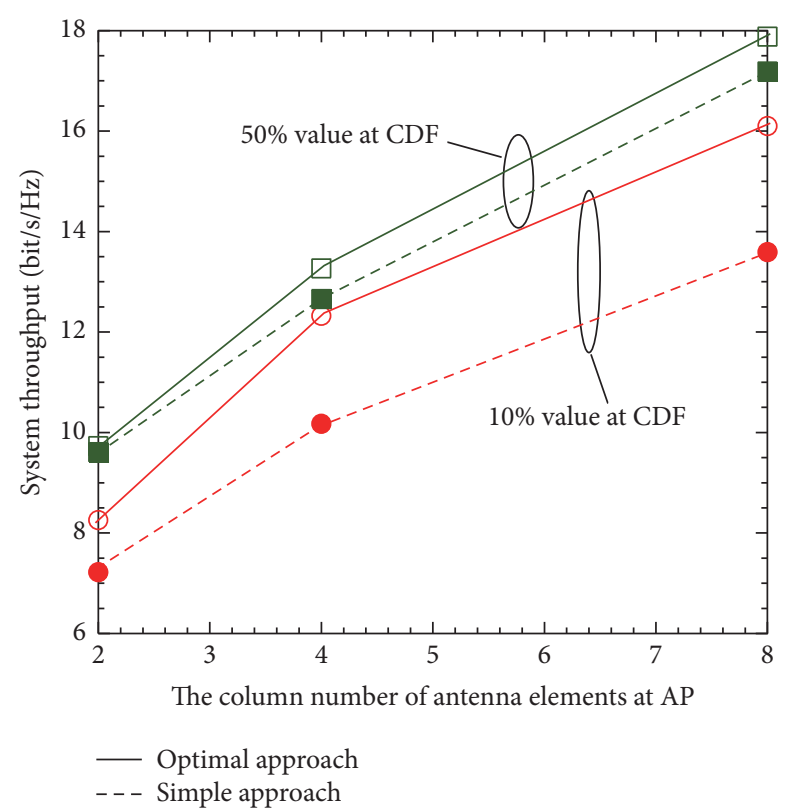

FIgURE 13: System throughput versus the number of AP antenna elements of vertical column.

matrices measured in our testbed of an office environment. The measurement results showed that the proposed scheme achieves higher system throughput, up to $30 \%$, than just power control even in indoor office environments.

In future work, we will evaluate the proposed scheme in non-line-of-sight (NLOS) environments including the effect of people in order to expand the application area.

\section{Conflicts of Interest}

The authors declare that there are no conflicts of interest regarding the publication of this paper.

\section{References}

[1] F. Rebecchi, M. Dias de Amorim, V. Conan, A. Passarella, R. Bruno, and M. Conti, "Data offloading techniques in cellular networks: a survey," IEEE Communications Surveys \& Tutorials, vol. 17, no. 2, pp. 580-603, 2015.

[2] A. Aijaz, H. Aghvami, and M. Amani, "A survey on mobile data offloading: technical and business perspectives," IEEE Wireless Communications, vol. 20, no. 2, pp. 104-112, 2013.

[3] K. Samdanis, T. Taleb, and S. Schmid, "Traffic offload enhancements for eUTRAN," IEEE Communications Surveys and Tutorials, vol. 14, no. 3, pp. 884-896, 2012.

[4] A. Damnjanovic, J. Montojo, Y. Wei et al., "A survey on 3GPP heterogeneous networks," IEEE Wireless Communications, vol. 18, no. 3, pp. 10-21, 2011.

[5] W. Lei, W. Hai, Y. Yinghui, and Z. Fei, "Heterogeneous network in LTE-Advanced system," in Proceedings of 12th IEEE International Conference on Communication Systems 2010, ICCS 2010, pp. 156-160, Singapore, Singapore, November 2010.

[6] R. Ferrus, O. Sallent, and R. Agusti, "Interworking in heterogeneous wireless networks: Comprehensive framework and future trends," IEEE Wireless Communications, vol. 17, no. 2, pp. 22-31, 2010.

[7] J. G. Andrews, "Seven ways that hetnets are a cellular paradigm shift," IEEE Communications Magazine, vol. 51, no. 3, pp. 136144, 2013.

[8] I. Hwang, B. Song, and S. Soliman, "A holistic view on hyperdense heterogeneous and small cell networks," IEEE Communications Magazine, vol. 51, no. 6, pp. 20-27, 2013.

[9] M. Yu, H. Luo, and K. K. Leung, "A dynamic radio resource management technique for multiple APs in WLANs," IEEE Transactions on Wireless Communications, vol. 5, no. 7, pp. 19101919, 2006.

[10] D. Gesbert, S. Hanly, H. Huang, S. Shamai Shitz, O. Simeone, and W. Yu, "Multi-cell MIMO cooperative networks: a new look at interference," IEEE Journal on Selected Areas in Communications, vol. 28, no. 9, pp. 1380-1408, 2010.

[11] G. Boudreau, J. Panicker, N. Guo, R. Chang, N. Wang, and S. Vrzic, "Interference coordination and cancellation for $4 \mathrm{G}$ networks," IEEE Communications Magazine, vol. 47, no. 4, pp. 74-81, 2009.

[12] V. P. Mhatre, K. Papagiannaki, and F. Baccelli, "Interference mitigation through power control in high density 802.11 WLANs," in Proceedings of the 26th IEEE International Conference on Computer Communications (INFOCOM '07), pp. 535-543, Barcelona, Spain, May 2007.

[13] A. Tölli, H. Pennanen, and P. Komulainen, "Distributed implementation of coordinated multi-cell beamforming," in Proceedings of 2009 IEEE 20th Personal, Indoor and Mobile Radio Communications Symposium, PIMRC 2009, Tokyo, Japan, September 2009.

[14] R. Kudo, Y. Takatori, K. Nishimori et al., "Spatial domain resource sharing for overlapping cells in indoor environment," International Journal of Digital Multimedia Broadcasting, vol. 2010, Article ID 642542, 2010. 
[15] B. Badic, T. O'Farrell, P. Loskot, and J. He, "Effect of the base station antenna beam tilting on energy consumption in cellular networks," in Proceedings of 2010 IEEE 72nd Vehicular Technology Conference Fall, VTC2010-Fall, Ottawa, ON, Canada, September 2010.

[16] X. Lu, A. Tölli, O. Piirainen, M. Juntti, and W. Li, "Comparison of antenna arrays in a 3-D multiuser multicell network," in Proceedings of the IEEE International Conference on Communications (ICC '11), pp. 1-6, June 2011.

[17] Q. Kuang, J. Speidel, and H. Droste, "Joint base-station association, channel assignment, beamforming and power control in heterogeneous networks," in Proceedings of IEEE 75th Vehicular Technology Conference, VTC Spring 2012, Yokohama, Japan, June 2012.

[18] F. Gholam, J. Vía, and I. Santamaría, "Beamforming design for simplified analog antenna combining architectures," IEEE Transactions on Vehicular Technology, vol. 60, no. 5, pp. 23732378, 2011.

[19] D. H. Pham, J. Gao, T. Tabata, H. Asato, S. Hori, and T. Wada, "Implementation of joint Pre-FFT adaptive array antenna and Post-FFT space diversity combining for mobile ISDB-T receiver," IEICE Transactions on Communications, vol. E91-B, no. 1, pp. 127-138, 2008.

[20] J. Nsenga, A. Bourdoux, and F. Horlin, "Mixed analog/digital beamforming for $60 \mathrm{GHz}$ MIMO frequency selective channels," in Proceedings of 2010 IEEE International Conference on Communications, ICC 2010, Cape Town, South Africa, South Africa, May 2010.

[21] D. Uchida and H. Arai, "Design of a MIMO antenna to enhance channel capacity for indoor base stations," in Proceedings of Joint 2012 IEEE International Symposium on Antennas and Propagation and USNC-URSI National Radio Science Meeting, APSURSI 2012, Chicago, IL, USA, July 2012.

[22] T. Murakami, R. Kudo, K. Ishihara, N. Honma, and M. Mizoguchi, "Cooperative interference management by beam tilt and power controls in an indoor multi-cell environment," in Proceedings of 2013 7th European Conference on Antennas and Propagation, EuCAP 2013, pp. 643-647, Gothenburg, Sweden, April 2013.

[23] K. Nishimori, R. Kudo, N. Honma, Y. Takatori, and M. Mizoguchi, "16×16 multiuser MIMO testbed employing simple adaptive modulation scheme," in Proceedings of VTC Spring 2009 - IEEE 69th Vehicular Technology Conference, Barcelona, Spain, April 2009. 


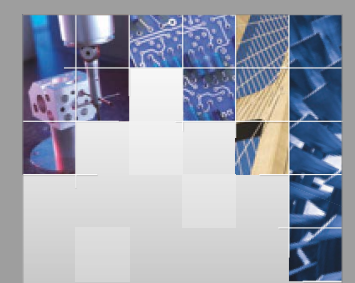

\section{Enfincering}
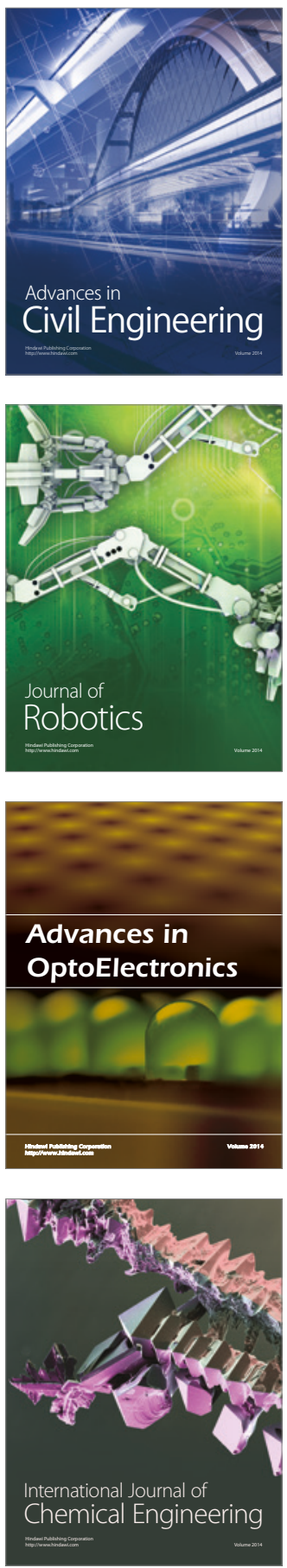

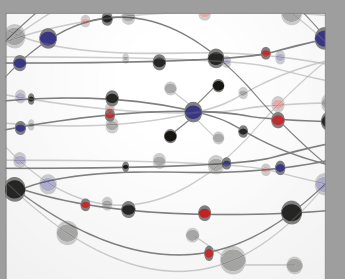

The Scientific World Journal

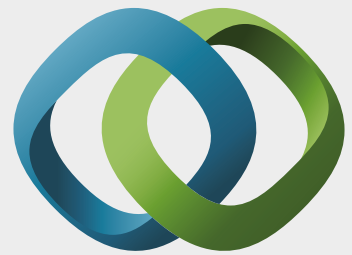

\section{Hindawi}

Submit your manuscripts at

https://www.hindawi.com
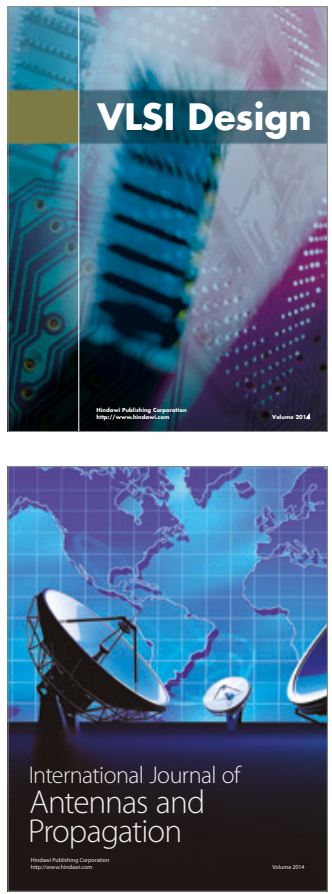

\section{Rotating}

Machinery
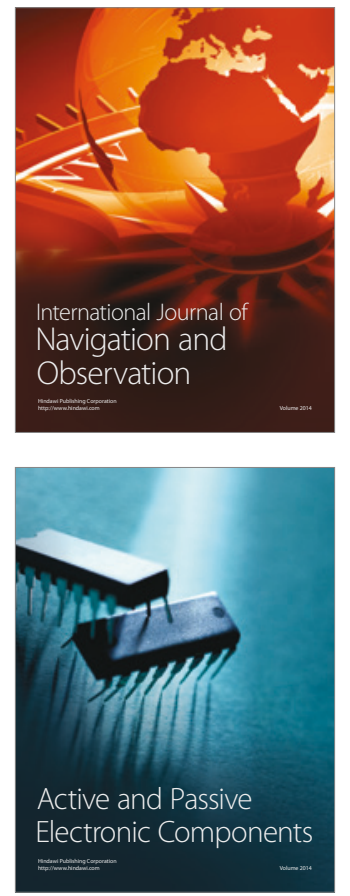
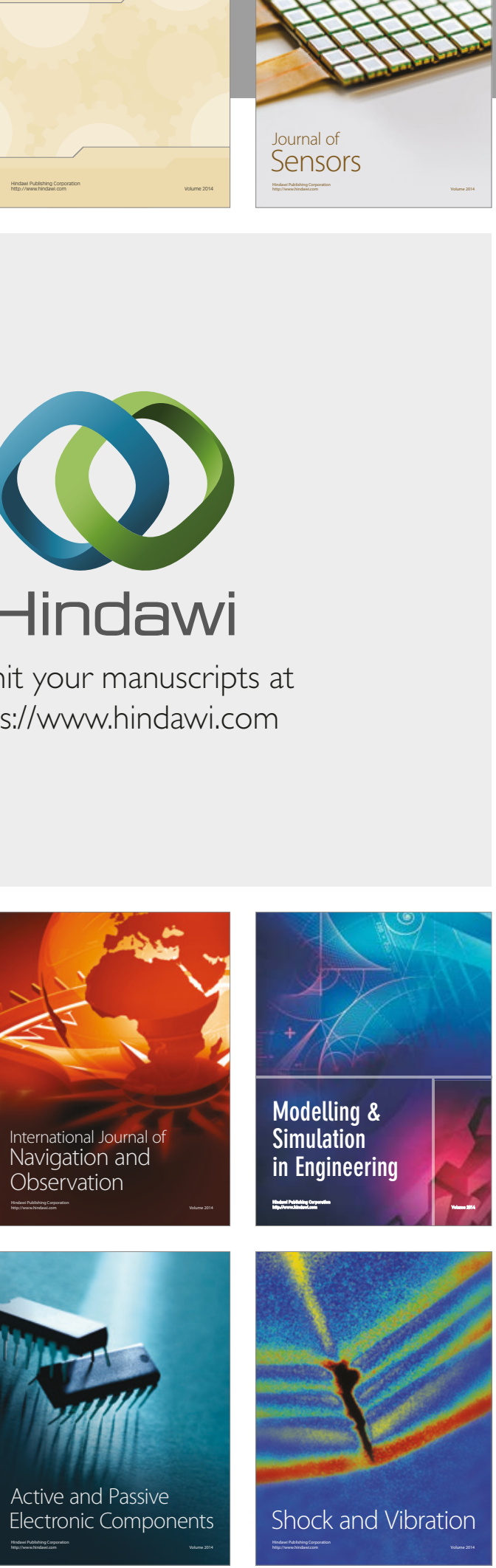
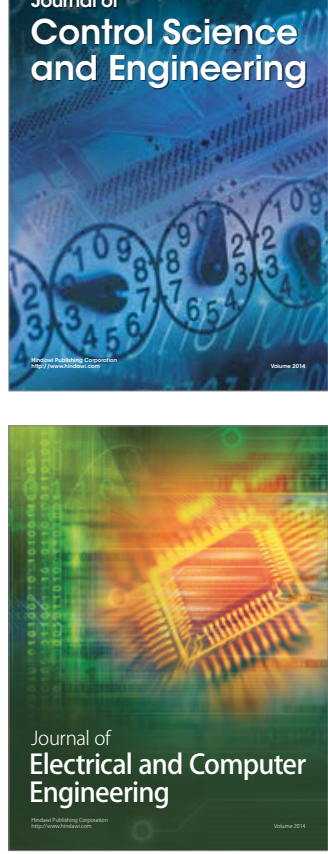

Distributed

Journal of

Control Science

and Engineering
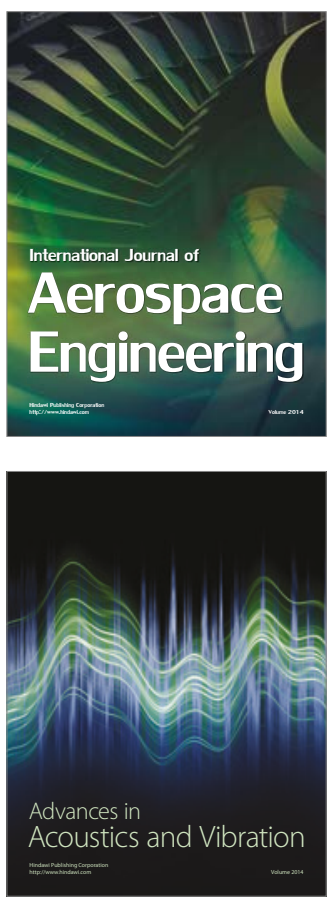

Sensor Networks 